\title{
Unexpectedly Lethal Hemothorax in the ICU
}

\section{Sakir Akin*, Corstiaan A den Uil, Dinis Dos Reis Miranda and Robert J van Thiel}

Department of Cardiology, Intensive Care Unit and Thoraxcenter, Erasmus Medical Center, Rotterdam, The Netherlands

\begin{abstract}
Chest-tube placement is one of the most common procedures performed to evacuate pleural effusion. Several types and sizes of chest tubes are available. Although chest-tube placement is straightforward, it may be associated with significant morbidity and mortality. We describe here a hemothorax in a patient with Veno-venous extra corporeal membrane oxygenator (VV-ECMO) which was treated with chest-tube placements by Seldinger technique.

VV-ECMO is increasingly being used as a bridge to recovery or transplantation in patients with severe pulmonary disease. Unexpected complications, like a hemothorax can cause severe hemodynamic derangement. Treatment of this complication remains challenging for several reasons in patients assisted by VV-ECMO. Introduction of a chesttube should therefore be regarded as a high risk intervention in VV-ECMO patients.
\end{abstract}

Keywords: Hemothorax; Chest tube; Seldinger technique; VVECMO

\section{Case Report}

A 69-year-old male was referred to our tertiary referral center. He had respiratory failure due to Legionella pneumonia. He had a history of previous pneumonia, superficial malignant neoplasm of bladder treated with TUR (transurethral resection), trans urethral resection of a papilloma, hypertension, percutaneous transluminal angioplasty of the femoral arteries, percutaneous coronary intervention of the ramus circumflexus, and coronary artery bypass grafting (CABG) eight years earlier. Drugs which he was taking at home were acetylsalicylic acid 100 mg q.d., rosuvastatine $20 \mathrm{mg}$ q.d., enalapril $20 \mathrm{mg}$ q.d., chloorthalidon $12,4 \mathrm{mg}$ q.d., amlodipine $5 \mathrm{mg}$ q.d. and tiotropium $18 \mu$ g q.d. He was admitted elsewhere five days earlier with progressive respiratory failure. The urine antigen tests for Legionella were positive, while aerobic and anaerobic cultures remained negative, after which he was started on ciprofloxacin $400 \mathrm{mg}$ b.i.d. doses adapted to his kidney function, and ceftriaxone $2000 \mathrm{mg}$ q.d. Because of increasingly difficult ventilation he was transferred to the ICU and intubated. Even ventilating in prone position didn't improve his situation and the patient was transferred to our center for placement of VV-ECMO [1-3]. Due to a pneumothorax shortly before transfer a chest-tube was placed in the referring hospital. At our ICU swift and uncomplicated cannulation of the right jugular and right femoral vein was performed. After this procedure we started with VV-ECMO at 5 liter/minute blood flow, 6 liter/minute sweep gas flow and at a rate of $2975 \mathrm{rpm}$ (PLS, MAQUET Cardiopulmonary AG, Rastatt). Cultures were taken and the antibiotic regime was changed to Levofloxacin $500 \mathrm{mg}$ intravenous (i.v.) q.d. adapted to his reduced kidney function, Cefotaxime $1000 \mathrm{mg}$ i.v. q.i.d. and selective digestive decontamination (SDD). Patient had already been started on continuous renal replacement therapy (CRRT). During the first hours the patient developed a second ipsilateral hemothorax that was not sufficiently drained by the first chest-tube, as it had become dislocated, which was the reason to place a second one, using a Seldinger technique.

The latter produced no fluid, and the patient became hemodynamically unstable, urgent new chest-tube of the same type (Thal-Quick Chest Tube Set, Cook Medical, Bloomington, USA) insertion with release of $300 \mathrm{ml}$ old blood was followed by emergency Computed tomography angiography (CTA). At that moment there was a hemoglobin value of $4.2 \mathrm{mmol} / \mathrm{L}(6,8 \mathrm{gr} / \mathrm{dl})$ with ongoing transfusion of erythrocytes. After cannulation we had omitted the use of heparin as we feared bleeding complications. CTA of the thorax (Figure 1) demonstrated contrast extravasation at distal end of the last chesttube. The blush was likely to come from a sub-segmental pulmonary

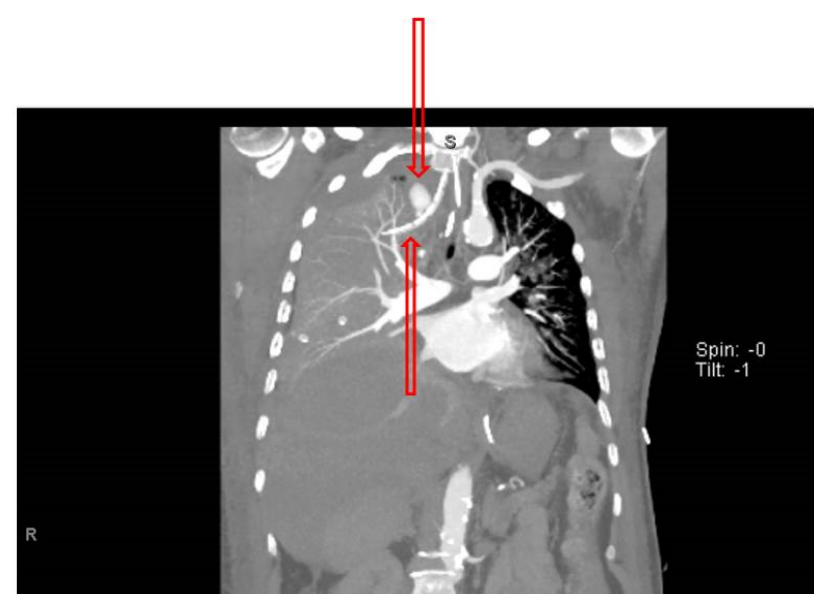

Figure 1: Computed tomography thorax: ECMO cannula through the superior vena cava and inferior vena cava in situ. Arterial blushes at the location of the basal chest-tube in which a curl is located and which can be determined or not properly be interfissural intrapulmonary lies. Active arterial extravasation at the location of the distal portion of the chest-tube. These seem to come from some sub-segmental branches of the middle lobe. Extensive hemothorax with substantial amount of blood basement and to a lesser degree apical right. Slight right ventral pneumothorax.

artery branch of the upper lung segment artery. This strongly suggested that the last chest-tube had caused the bleeding. Following this the patient underwent an angiography (Figure 2) and coiling procedure. Interestingly the contrast fluid stopped draining from the chest-tube after the coiling procedure. The next day the patient was taken to theater for surgical evacuation of retained blood clots during which procedure minor additional bleeding was corrected.

Unfortunately the bleeding continued despite adequate blood

${ }^{*}$ Corresponding author: Sakir Akin, Department of Cardiology, Intensive Care Unit and Thoraxcenter, Erasmus Medical Center, Rotterdam, The Netherlands, Tel: +31 10703 3276; E-mail: s.akin@erasmusmc.nl

Received April 05, 2016; Accepted May 23, 2016; Published May 26, 2016

Citation: Akin S, den Uil CA, dos Reis Miranda D, van Thiel RJ (2016) Unexpectedly Lethal Hemothorax on the ICU. J Pulm Respir Med 6: 349. doi:10.4172/2161105X.1000349

Copyright: (c) 2016 Akin S, et al. This is an open-access article distributed under the terms of the Creative Commons Attribution License, which permits unrestricted use, distribution, and reproduction in any medium, provided the original author and source are credited. 


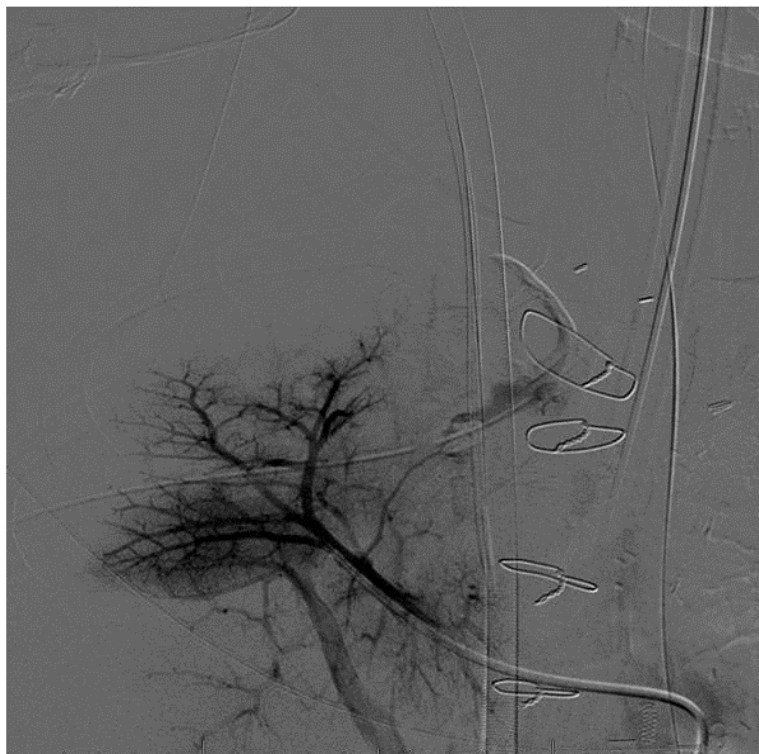

Figure 2. Pulmonary angiogram pre-coiling with chest-tubes in situ first blush but unlike CT, only upper chest-tube; large extravasation from a spastic pulmonary side branch.

product supplementation. Cardiac arrest interrupted transportation for a repeat surgical intervention, and proved irreversible.

\section{Discussion}

The Seldinger technique is known for its very low complication rate since its introduction. The desired cavity or vessel is punctured with a sharp hollow needle (trocar) and a round-tipped guidewire is then advanced through the lumen of the trocar, and the trocar is withdrawn [4]. Inherent to this sharp puncture it may lead to hemorrhage or perforation of the organ in question. Loss of the guidewire into the cavity or blood vessel is a generally preventable complication. These steps could be guided by ultrasound, fluoroscopy or computed tomography for the direction of the wire, sheath, catheter or chest-tube. After passing a sheath or tube, the guidewire is withdrawn. A chesttube has been passed over the guidewire into the right thorax in our case. Angiography and the field on interventional radiology became relatively risk free after introduction of Seldinger technique. Use of this technique guided by computed tomography could prevent hemothorax in (vulnerable) patients with pneumothorax or pleural effusion [5].

Our patient presented with respiratory failure due to Legionella pneumonia complicated by pneumothorax and was admitted for implantation of VV-ECMO. Cannulation was uncomplicated. $\mathrm{He}$ subsequently developed hemothorax probably due to the first chesttube and aggravated by heparin use for cannulation. The second and third chest-tube placements by Seldinger technique aggravated bleeding, necessitating further investigation (Figure 1). A Seldinger technique has the big advantages of smaller incision, theoretically reducing bleeding risk, minimal tissue dissection resulting in less pain for the patient, and a more aesthetic scar after removal of the tube. But there is an inability to digitally manipulate the pleural space and limited ability to direct the tube to an exact location in the pleural space and the tube will follow where the guide wire has gone.

From the literature we know the importance of an emergency CTA scan in such patients on ECMO [5]. The patient underwent a CT-scan of the thorax by which an active bleeding was diagnosed (Figure 1), and ultimately underwent a percutaneous coiling of a side branch of pulmonic artery (Figure 2). The relationship between the third chesttube and the bleeding was not clear. However we decided not to remove the chest-tube which should seep The relationship between the chesttube and the bleeding was not clear. But it seems likely that the tip of this chest-tube was responsible for local injury, reason why we didn't remove it until the surgical intervention. The next day he underwent a lateral thoracotomy with evacuation of accumulated blood clots amounting 3 liters. After further correction of coagulopathy including abstinence of heparin, the patient stabilized for a while. Unfortunately he deteriorated due to re-bleeding and died before a second thoracotomy could be performed. All the time the ECMO system functioned well with 5,1 liter blood-flow and 4,5 liter sweep-flow. It was a challenge to stop intrathoracic bleeding with coiling during support on VV-ECMO. Refractory hypovolemic shock was aborted for a while by coiling of a bleeding sub-segmental pulmonary artery and subsequent surgical intervention.

Our message is that hemothorax in ECMO patients can be caused easily. Furthermore the minimal invasiveness of the chest-tube system used did not result in lower bleeding risk. We suggest being careful with use of Seldinger introduction of chest-tubes in these vulnerable patients and sticking with the conventional surgical way if there is no option for a saver method of CT guided percutaneous drainage.

This case report emphasizes a fatal complication of chest tube placement in a patient with vulnerable lungs on ECMO. Although the Seldinger technique for chest-tube placement is growing in popularity and we have shown that at least extreme caution is necessary when using this technique in VV-ECMO patients with very vulnerable lungs.

\section{Références}

1. Mahmood K, Wahidi MM (2013) Straightening out chest tubes: what size, what type, and when. Clin Chest Med 34: 63-71.

2. Zwischenberger JB, Cilley RE, Hirschl RB, Heiss KF, Conti VR, et al. (1988) Lifethreatening intrathoracic complications during treatment with extracorporeal membrane oxygenation. J Pediatr Surg 23: 599-604.

3. Rigby MR, Kamat P, Vats A, Heard M (2013) Controlling intrathoracic hemorrhage on ECMO: help from Factor VIla and Virchow. Perfusion 28: 201206.

4. Seldinger SI (1953) Catheter replacement of the needle in percutaneous arteriography; a new technique. Acta radiol 39: 368-376.

5. Lidegran MK, Ringertz HG, Frenckner BP, Linden VB (2005) Chest and abdominal CT during extracorporeal membrane oxygenation: Clinical benefits in diagnosis and treatment. Acad Radiol 12: 276-285. 